\title{
Research Article \\ Fixed Point Theorems for a Weaker Meir-Keeler Type $\psi$-Set Contraction in Metric Spaces
}

\author{
Chi-Ming Chen and Tong-Huei Chang \\ Department of Applied Mathematics, National Hsinchu University of Education, Hsinchu 300, Taiwan \\ Correspondence should be addressed to Tong-Huei Chang, thchang@mail.nhcue.edu.tw
}

Received 25 March 2009; Accepted 19 June 2009

Recommended by Marlene Frigon

We define a weaker Meir-Keeler type function and establish the fixed point theorems for a weaker Meir-Keeler type $\psi$-set contraction in metric spaces.

Copyright (C 2009 C.-M. Chen and T.-H. Chang. This is an open access article distributed under the Creative Commons Attribution License, which permits unrestricted use, distribution, and reproduction in any medium, provided the original work is properly cited.

\section{Introduction and Preliminarie}

In 1929, Knaster et al. [1] had proved the well-known KKM theorem on $n$-simplex. Besides, in 1961, Fan [2] had generalized the KKM theorem to an infinite dimensional topological vector space. Later, Amini et al. [3] had introduced the class of KKM-type mappings on metric spaces and established some fixed point theorems for this class. In this paper, we define a weaker Meir-Keeler type function and establish the fixed point theorems for a weaker MeirKeeler type $\psi$-set contraction in metric spaces.

Throughout this paper, by $\Re_{+}$we denote the set of all real nonnegative numbers, while $\mathbb{N}$ is the set of all natural numbers. We digress briefly to list some notations and review some definitions. Let $X$ and $Y$ be two Hausdorff topological spaces, and let $T: X \rightarrow 2^{Y}$ be a setvalued mapping. Then $T$ is said to be closed if its graph $\mathcal{G}_{T}=\{(x, y) \in X \times Y: y \in T(x)\}$ is closed. $T$ is said to be compact if the image $T(X)$ of $X$ under $T$ is contained in a compact subset of $Y$. If $D$ is a nonempty subset of $X$, then $\langle D\rangle$ denotes the class of all nonempty finite subsets of $D$. And, the following notations are used:

(i) $T(x)=\{y \in Y: y \in T(x)\}$,

(ii) $T(A)=\cup_{x \in A} T(x)$,

(iii) $T^{-1}(y)=\{x \in X: y \in T(x)\}$, and

(iv) $T^{-1}(B)=\{x \in X: T(x) \cap B \neq \phi\}$. 
Let $(M, d)$ be a metric space, $X \subset M$ and $\delta>0$. Let $B_{M}(X, \delta)=\{x \in M: d(x, X) \leqslant \delta\}$, and let $N_{M}(X, \delta)=\{x \in M: d(x, X)<\delta\}$. following

Suppose that $X$ is a bounded subset of a metric space $(M, d)$. Then we define the

(i) $\operatorname{co}(X)=\cap\{B \subset M: B$ is a closed ball in $M$ such that $X \subset B\}$, and

(ii) $X$ is said to be subadmissible [3], if for each $A \in\langle X\rangle, \operatorname{co}(A) \subset X$.

In 1996, Chang and Yen [4] introduced the family $\operatorname{KKM}(X, Y)$ on the topological vector spaces and got results about fixed point theorems, coincidence theorems, and its applications on this family. Later, Amini et al. [3] introduced the following concept of the $\operatorname{KKM}(X, Y)$ property on a subadmissible subset of a metric space $(M, d)$.

Let $X$ be an nonempty subadmissible subset of a metric space $(M, d)$, and let $Y$ a topological space. If $T, F: X \rightarrow 2^{Y}$ are two set-valued mappings such that for any $A \in\langle X\rangle$, $T(c o(A)) \subset F(A)$, then $F$ is called a generalized KKM mapping with respect to $T$. If the set-valued mapping $T: X \rightarrow 2^{Y}$ satisfies the requirement that for any generalized KKM mapping $F$ with respest to $T$, the family $\{\overline{F(x)}: x \in X\}$ has finite intersection property, then $\mathrm{T}$ is said to have the $\mathrm{KKM}$ property. The class $\operatorname{KKM}(X, Y)$ is denoted to be the set $\{T: X \rightarrow$ $2^{Y}: T$ has the KKM property\}.

Recall the notion of the Meir-Keeler type function. A function $\psi: \mathfrak{R}_{+} \rightarrow \mathfrak{R}_{+}$is said to be a Meir-Keeler type function (see [5]), if for each $\eta \in \mathfrak{R}_{+}$, there exists $\delta=\delta(\eta)>0$ such that for $t \in \mathfrak{R}_{+}$with $\eta \leq t<\eta+\delta$, we have $\psi(t)<\eta$.

We now define a new weaker Meir-Keeler type function as follows.

Definition 1.1. We call $\psi: \mathfrak{R}_{+} \rightarrow \mathfrak{R}_{+}$a weaker Meir-Keeler type function, if for each $\eta>0$, there exists $\delta>0$ such that for $t \in \mathfrak{R}_{+}$with $\eta \leq t<\eta+\delta$, and there exists $n_{0} \in \mathbb{N}$ such that $\psi^{n_{0}}(t)<\eta$.

A function $\psi: \mathfrak{R}_{+} \rightarrow \mathfrak{R}_{+}$is said to be upper semicontinuous, if for each $t_{0} \in \mathfrak{R}_{+}$, $\lim _{t \rightarrow t_{0}} \sup \psi(t) \leq \psi\left(t_{0}\right)$. Recall also that $\psi: \mathfrak{R}_{+} \rightarrow \mathfrak{R}_{+}$is said to be a comparison function (see [6]) if it is increasing and $\lim _{n \rightarrow \infty} \psi^{n}(t)=0$. As a consequence, we also have that for each $t>0, \psi(t)<t$, and $\psi(0)=0, \psi$ is continuous at 0 . We generalize the comparison function to be the other form, as follows.

Definition 1.2. We call $\psi: \mathfrak{R}_{+} \rightarrow \mathfrak{R}_{+}$a generalized comparison function, if $\psi$ is upper semicontinuous with $\psi(0)=0$ and $\psi(t)<t$ for all $t>0$.

Proposition 1.3. If $\psi: \mathfrak{R}_{+} \rightarrow \mathfrak{R}_{+}$is a generalized comparison function, then there exists a strictly increasing, continuous function $\alpha: \mathfrak{R}_{+} \rightarrow \mathfrak{R}_{+}$such that $\psi(t) \leq \alpha(t)<t$, for all $t>0$.

Proof. Let $\phi(t)=t-\psi(t)$. Since $\psi: \mathfrak{R}_{+} \rightarrow \mathfrak{R}_{+}$is an upper semicontinuous function, hence it attains its minimum in any closed bounded interval of $\Re_{+}$.

For each $n \in \mathbb{N}$, we first define four sequences $\left\{a_{n}\right\},\left\{b_{n}\right\},\left\{c_{n}\right\}$, and $\left\{d_{n}\right\}$ as follows:

(i) $a_{n}=\min _{t \in[n, n+1]} \phi(t)$,

(ii) $b_{n}=\min _{t \in[1 /(n+1), 1 / n]} \phi(t)$,

(iii) $c_{1}, d_{1}=\min \left\{a_{1}, b_{1}\right\}$,

(iv) $c_{n}=\min \left\{c_{1}, a_{1}, a_{2}, \ldots, a_{n}\right\}$ for $n \geq 2$, and

(v) $d_{n}=\min \left\{c_{1}, b_{1}, b_{2}, \ldots, b_{n}, 1 / n(n+1)\right\}$ for $n \geq 2$. 
Fixed Point Theory and Applications

And, we next let a function $\alpha: \mathfrak{R}_{+} \rightarrow \mathfrak{R}_{+}$satisfy the following:

(1) $\alpha(0)=0, \alpha(n)=n-c_{n}, \alpha(1 / n)=1 / n-d_{n}$,

(2) if $n \leq t \leq n+1$, then

$$
\alpha(t)=(t-n) \alpha(n+1)+(n+1-t) \alpha(n),
$$

(3) if $1 /(n+1) \leq t \leq 1 / n$, then

$$
\alpha(t)=\alpha\left(\frac{1}{n+1}\right)+n(n+1)\left[\alpha\left(\frac{1}{n}\right)-\alpha\left(\frac{1}{n+1}\right)\right]\left(t-\frac{1}{n+1}\right) .
$$

Then by the definition of the function $\alpha$, we are easy to conclude that $\alpha$ is strictly increasing, continuous. We complete the proof by showing that $\psi(t) \leq \alpha(t)$ for all $t>0$.

If $n \leq t \leq n+1$, then

$$
\begin{aligned}
\alpha(t) & =(t-n) \alpha(n+1)+(n+1-t) \alpha(n) \\
& =\left(t-c_{n}\right)+(t-n)\left(c_{n}-c_{n+1}\right) \\
& \geq t-[t-\psi(t)]+(t-n)\left(c_{n}-c_{n+1}\right) \\
& \geq \psi(t) .
\end{aligned}
$$

If $1 /(n+1) \leq t \leq 1 / n$, then

$$
\begin{aligned}
\alpha(t) & =\alpha\left(\frac{1}{n+1}\right)+n(n+1)\left[\alpha\left(\frac{1}{n}\right)-\alpha\left(\frac{1}{n+1}\right)\right]\left(t-\frac{1}{n+1}\right) \\
& =t-d_{n}+\left(d_{n}-d_{n+1}\right)[(n+1)-n(n+1) t] \\
& \geq t-[t-\psi(t)]+\left(d_{n}-d_{n+1}\right)[(n+1)-n(n+1) t] \\
& \geq \psi(t) .
\end{aligned}
$$

So $\psi(t) \leq \alpha(t)$ for all $t>0$.

Since $\alpha(n)<n$ and $\alpha(1 / n)<1 / n$ for all $n \in \mathbb{N}$, so $\alpha(t)<t$ for all $t>0$.

Proposition 1.4. If $\psi: \mathfrak{R}_{+} \rightarrow \mathfrak{R}_{+}$is a generalized comparison function, then there exists a strictly increasing, continuous function $\alpha: \mathfrak{R}_{+} \rightarrow \mathfrak{R}_{+}$such that

$$
\begin{gathered}
\psi(t) \leq \alpha(t)<t, \quad \text { for all } t>0 \\
\lim _{t \rightarrow \infty} \alpha(t)=\infty
\end{gathered}
$$

Proof. By Proposition 1.3, there exists a strictly increasing, continuous function $\bar{\alpha}: \mathfrak{R}_{+} \rightarrow \mathfrak{R}_{+}$ such that $\psi(t) \leq \bar{\alpha}(t)$, for all $t>0$. So, we may assume that $\lim _{t \rightarrow \infty} \alpha(t)=\infty$, by letting $\alpha(t)=(\bar{\alpha}(t)+t) / 2$ for all $t \in \mathfrak{R}_{+}$. 
Remark 1.5. In the above case, the function $\alpha$ is invertible. If for each $t>0$, we let $\alpha^{0}(t)=$ $t$ and $\alpha^{-n}(t)=\alpha^{-1}\left(\alpha^{-n+1}(t)\right)$ for all $n \in \mathbb{N}$, then we have that $\lim _{n \rightarrow \infty} \alpha^{-n}(t)=\infty$; that is, $\lim _{n \rightarrow \infty} \alpha^{n}(t)=0$.

Proof. We claim that $\lim _{n \rightarrow \infty} \alpha^{n}(t)=0$, for $t>0$. Suppose that $\lim _{n \rightarrow \infty} \alpha^{-n}(t)=\eta$ for some positive real number $\eta$. Then

$$
\eta=\lim _{n \rightarrow \infty} \alpha^{-n}(t)=\alpha^{-1}\left(\lim _{n \rightarrow \infty} \alpha^{-n+1}(t)\right)=\alpha^{-1}(\eta)>\eta
$$

which is a contradiction. So $\lim _{n \rightarrow \infty} \alpha^{n}(t)=0$.

We now are going to give the axiomatic definition for the measure of noncompactness in a complete metric space.

Definition 1.6. Let $(M, d)$ be a metric space, and let $B(M)$ the family of bounded subsets of M. A map

$$
\Phi: B(M) \rightarrow[0, \infty)
$$

is called a measure of noncompactness defined on $M$ if it satisfies the following properties:

(i) $\Phi\left(D_{1}\right)=0$ if and only if $D_{1}$ is precompact, for each $D_{1} \in B(M)$,

(ii) $\Phi\left(\overline{D_{1}}\right)=\Phi\left(D_{1}\right)$, for each $D_{1} \in B(M)$,

(iii) $\Phi\left(D_{1} \cup D_{2}\right)=\max \left\{\Phi\left(D_{1}\right), \Phi\left(D_{2}\right)\right\}$, for each $D_{1}, D_{2} \in B(M)$,

(iv) $\Phi\left(D_{1}\right)=\Phi\left(\operatorname{co}\left(D_{1}\right)\right)$, for each $D_{1} \in B(M)$.

The above notion is a generalization of the set measure of noncompactness in metric spaces. The following $\alpha$-measure is a well-known measure of noncompactness.

Definition 1.7. Let $(M, d)$ be a complete metric space, and let $B(M)$ the family of bounded subsets of $M$. For each $D \in B(M)$, we define the set measure of noncompactness $\alpha(D)$ by:

$$
\alpha(D)=\inf \{\varepsilon>0: D \text { can be covered by finitely many sets with diameter } \leqslant \varepsilon\} .
$$

Definition 1.8. Let $X$ be a nonempty subset of a metric space $(M, d)$. If a mapping $T: X \rightarrow 2^{M}$ with for each $A \subset X, A$ and $T(A)$ are bounded, then $T$ is called

(i) a $k$-set contraction, if for each $A \subset X, \alpha(T(A)) \leq k \alpha(A)$, where $k \in[0,1)$,

(ii) a weaker Meir-Keeler type $\psi$-set contraction, if for each $A \subset X, \alpha(T(A)) \leq \psi(\alpha(A))$, where $\psi: \mathfrak{R}_{+} \rightarrow \mathfrak{R}_{+}$is a weaker Meir-Keeler type function,

(iii) a generalized comparison (comparison) type $\psi$-set contraction, if for each $A \subset X$, $\alpha(T(A)) \leq \psi(\alpha(A))$, where $\psi: \mathfrak{R}_{+} \rightarrow \mathfrak{R}_{+}$is a generalized comparison (comparison) function.

Remark 1.9. It is clear that if $T: X \rightarrow 2^{M}$ is a $k$-set contraction, then $T$ is a weaker Meir-Keeler type $\psi$-set contraction, but the converse does not hold. 


\section{Main Results}

Using the conception of the weaker Meir-Keeler type function, we establish the following important theorem.

Theorem 2.1. Let $X$ be a nonempty bounded subadmissible subset of a metric space $(M, d)$. If $T: X \rightarrow 2^{X}$ is a weaker Meir-Keeler type $\psi$-set contraction with for each $t \in \mathfrak{R}_{+},\left\{\psi^{n}(t)\right\}_{n \in \mathbb{N}}$ is nonicreasing, then $X$ contains a precompact subadmissible subset $K$ with $T(K) \subset K$.

Proof. Take $y \in X$, and let

$$
\begin{gathered}
X_{0}=X, \quad X_{1}=\operatorname{co}\left(T\left(X_{0}\right) \cup\{y\}\right), \\
X_{n+1}=c o\left(T\left(X_{n}\right) \cup\{y\}\right), \quad \text { for each } n \in N .
\end{gathered}
$$

Then

(1) $X_{n}$ is a subadmissible subset of $X$, for each $n \in N$;

(2) $T\left(X_{n}\right) \subset X_{n+1} \subset X_{n}$, for each $n \in N$.

Since $T: X \rightarrow 2^{X}$ is a weaker Meir-Keeler type $\psi$-set contraction, then $\alpha\left(T\left(X_{n}\right)\right) \leq$ $\psi\left(\alpha\left(X_{n}\right)\right)$ and $\alpha\left(X_{n+1}\right)=\alpha\left(\operatorname{co}\left(T\left(X_{n}\right) \cup\{y\}\right)\right) \leq \alpha\left(T\left(X_{n}\right)\right)$. Hence, we conclude that $\alpha\left(X_{n}\right) \leq$ $\psi^{n}(\alpha(X))$.

Since $\left\{\psi^{n}(\alpha(X))\right\}_{n \in \mathbb{N}}$ is nonincreasing, it must converge to some $\eta$ with $\eta \geq 0$; that is, $\lim _{n \rightarrow \infty} \psi^{n}(\alpha(X))=\eta \geq 0$. We now claim that $\eta=0$. On the contrary, assume that $\eta>$ 0 .Then by the definition of the weaker Meir-Keeler type function, there exists $\delta>0$ such that for each $A \subset X$ with $\eta \leq \alpha(A)<\eta+\delta$, there exists $n_{0} \in \mathbb{N}$ such that $\psi^{n_{0}}(\alpha(A))<\eta$. Since $\lim _{n \rightarrow \infty} \psi^{n}(\alpha(X))=\eta$, there exists $m_{0} \in \mathbb{N}$ such that $\eta \leq \psi^{m}(\alpha(X))<\eta+\delta$, for all $m \geq m_{0}$. Thus, we conclude that $\psi^{m_{0}+n_{0}}(\alpha(X))<\eta$. So we get a contradiction. So $\lim _{n \rightarrow \infty} \psi^{n}(\alpha(X))=0$, and so $\lim _{n \rightarrow \infty} \alpha\left(X_{n}\right)=0$.

Let $X_{\infty}=\cap_{n \in \mathbb{N}} X_{n}$. Then $X_{\infty}$ is a nonempty precompact subadmissible subset of $X$, and by (2), we have $T\left(X_{\infty}\right) \subset X_{\infty}$.

Remark 2.2. In the process of the proof of Theorem 2.1, we call the set $X_{\infty}$ a Meir-Keeler type precompact-inducing subadmissible subset of $X$. corollary.

Applying Proposition 1.3, 1.4, and Remark 1.5, we are easy to conclude the following

Corollary 2.3. Let $X$ be a nonempty bounded subadmissible subset of a metric space $(M, d)$. If $T: X \rightarrow 2^{X}$ is a generalized comparison (comparison) type $\psi$-set contraction, then $X$ contains a precompact subadmissible subset $K$ with $T(K) \subset K$.

Proof. The proof is similar to the proof of Theorem 2.1; we omit it.

Remark 2.4. In the process of the proof of Corollary 2.3, we also call the set $X_{\infty}$ a generalized comparison type precompact-inducing subadmissible subset of $X$.

Corollary 2.5. Let $X$ be a nonempty bounded subadmissible subset of a metric space $(M, d)$. If $T$ : $X \rightarrow 2^{X}$ is a $k$-set contraction, then $X$ contains a precompact subadmissible subset $K$ with $T(K) \subset K$. 
Following the concepts of the $\operatorname{KKM}(X, Y)$ family (see [3]), we immediately have the following Lemma 2.6.

Lemma 2.6. Let $X$ be a nonempty subadmissible subset of a metric space $(M, d)$, and let $Y$ a topological spaces. Then $\left.T\right|_{D} \in \operatorname{KKM}(D, Y)$, whenever $T \in K K M(X, Y)$, and $D$ is a nonempty subadmissible subset of $X$.

We now concern a fixed point theorem for a weaker Meir-Keeler type $\psi$-set contraction in a complete metric space, which needs not to be a compact map.

Theorem 2.7. Let $X$ be a nonempty bounded subadmissible subset of a metric space $(M, d)$. If $T \in$ $\operatorname{KKM}(X, X)$ is a weaker Meir-Keeler type $\psi$-set contraction with for each $t \in \mathfrak{R}_{+},\left\{\psi^{n}(t)\right\}_{n \in \mathbb{N}}$ is nonicreasing, and closed with $\overline{T(X)} \subset X$, then $T$ has a fixed point in $X$.

Proof. By the same process of Theorem 2.1, we get a weaker Meir-Keeler type precompactinducing subadmissible subset $X_{\infty}$ of $X$. Since $\overline{T(X)} \subset X$ and $T\left(X_{n+1}\right) \subset T\left(X_{n}\right) \subset T(X)$ for each $n \in N$, we have $\overline{T\left(X_{n+1}\right)} \subset \overline{T\left(X_{n}\right)} \subset X$ for each $n \in N$. Since $\alpha\left(\overline{T\left(X_{n}\right)}\right) \rightarrow 0$ as $n \rightarrow \infty$, by the above Lemma 2.6, we have that $\overline{T\left(X_{\infty}\right)}$ is a nonempty compact subset of $X$.

Since $T \in K K M(X, X)$ and $X_{\infty}$ is a nonempty subadmissible subset of $X$, by Lemma 2.6, $\left.T\right|_{X_{\infty}} \in K K M\left(X_{\infty}, X\right)$.

We now claim that for each $\varepsilon$, there exists an $x_{\varepsilon} \in X_{\infty}$ such that $B\left(x_{\varepsilon}, \varepsilon\right) \cap T\left(x_{\varepsilon}\right) \neq \phi$. If the above statement is not true, then there exists $\varepsilon^{\prime}$ such that $B\left(x, \varepsilon^{\prime}\right) \cap T(x)=\phi$, for all $x \in X_{\infty}$. Let $K=\overline{T\left(X_{\infty}\right)} \subset X$. Then we now define $F: X_{\infty} \rightarrow 2^{K}$ by

$$
F(x)=K \backslash N\left(x, \varepsilon^{\prime}\right), \quad \text { for each } x \in X_{\infty} .
$$

Then

(1) $F(x)$ is compact, for each $x \in X_{\infty}$, and

(2) $F$ is a generalized KKM mapping with respect to $\left.T\right|_{X_{\infty}}$.

We prove (2) by contradiction. Suppose $F$ is not a generalized KKM mapping with respect to $\left.T\right|_{X_{\infty}}$. Then there exists $A=\left\{x_{1}, x_{2}, \ldots, x_{n}\right\} \in\left\langle X_{\infty}\right\rangle$ such that

$$
T\left(\operatorname{co}\left\{x_{1}, x_{2}, \ldots, x_{n}\right\}\right) \nsubseteq \cup_{i=1}^{n} F\left(x_{i}\right)
$$

Choose $\mu \in \operatorname{co}\left\{x_{1}, x_{2}, \ldots, x_{n}\right\}$ and $v \in T(\mu) \subset \overline{T\left(X_{\infty}\right)}=K$ such that $v \notin \cup_{i=1}^{n} F\left(x_{i}\right)$. From the definition of $F$, it follows that $\nu \in N\left(x_{i}, \varepsilon^{\prime}\right)$, for each $i \in\{1,2, \ldots, n\}$. Since $\mu \in$ $\operatorname{co}\left\{x_{1}, x_{2}, \ldots, x_{n}\right\}, v \in T(\mu)$, we have $\mu \in \operatorname{co}(A) \subset B\left(\nu, \varepsilon^{\prime}\right)$, which implies that $v \in B\left(\mu, \varepsilon^{\prime}\right)$. Therefore, $v \in T(\mu) \cap B\left(\mu, \varepsilon^{\prime}\right)$. This contradicts to $T(\mu) \cap B\left(\mu, \varepsilon^{\prime}\right)=\phi$. Hence, $\mathrm{F}$ is a generalized KKM mapping with respect to $\left.T\right|_{X_{\infty}}$.

Since $\left.T\right|_{X_{\infty}} \in \operatorname{KKM}\left(X_{\infty}, X\right)$, the family $\left\{F(x): x \in X_{\infty}\right\}$ has the finite intersection property, and so we conclude that $\cap_{x \in X_{\infty}} F(x) \neq \phi$. Choose $\eta \in \cap_{x \in X_{\infty}} F(x)$, then $\eta \in K \backslash N\left(x, \varepsilon^{\prime}\right)$ for all $x \in X_{\infty}$. But, since $\eta \in \cap_{x \in X_{\infty}} F(x)$ and $K \subset \overline{X_{\infty}} \subset \cup_{x \in \bar{X}_{\infty}} N\left(x,(1 / 2) \varepsilon^{\prime}\right)$, so there exists an $x_{0} \in X_{\infty}$ such that $\eta \in N\left(x_{0}, \varepsilon^{\prime}\right)$. So, we have reached a contradiction.

Therefore, we have proved that for each $\varepsilon>0$, there exists an $x_{\varepsilon} \in X_{\infty}$ such that $B\left(x_{\varepsilon}, \varepsilon\right) \cap T\left(x_{\varepsilon}\right) \neq \phi$. Let $y_{\varepsilon} \in B\left(x_{\varepsilon}, \varepsilon\right) \cap T(\varepsilon)$. Since $y_{\varepsilon} \subset K$ and $K$ is compact, we may assume 
that $\left\{y_{\varepsilon}\right\}$ converges to some $\bar{y} \in K$, then $x_{\varepsilon}$ also converges to $\bar{y}$. Since $T$ is closed, we have $\bar{y} \in T(\bar{y})$. This completes the proof.

Corollary 2.8. Let $X$ be a nonempty bounded subadmissible subset of a metric space $(M, d)$. If $T \in$ $K K M(X, X)$ is a generalized composion type $\psi$-set contraction and closed with $\overline{T(X)} \subset X$, then $T$ has a fixed point in $X$.

Corollary 2.9. Let $X$ be a nonempty bounded subadmissible subset of a metric space $(M, d)$. If $T \in$ $\operatorname{KKM}(X, X)$ is a $k$-set contraction and closed with $\overline{T(X)} \subset X$, then $T$ has a fixed point in $X$.

The $\Phi$-spaces, in an abstract convex space setting, were introduced by Amini et al.[7]. An abstract convex space $(X, C)$ consists of a nonempty topological space $X$ and a family $C$ of subsets of $X$ such that $X$ and $\phi$ belong to $C$, and $C$ is closed under arbitrary intersection. Let $(X, \mathcal{C})$ be an abstract convex space, and let $Y$ a topological space. A map $T: Y \rightarrow 2^{X}$ is called a $\Phi$-mapping if there exists a multifunction $F: Y \rightarrow 2^{X}$ such that

(i) for each $y \in Y, A \in\langle F(y)\rangle$ implies $\operatorname{ad}_{\mathcal{C}}(A) \subset T(y)$;

(ii) $Y=\cup_{x \in X} \operatorname{int} F^{-1}(x)$.

The mapping $F$ is called a companion mapping of $T$. Furthermore, if the abstract convex space $(X, \mathcal{C})$ which has a uniformity $\mathcal{U}$ and $\mathcal{U}$ has an open symmetric base family $\mathbb{N}$, then $X$ is called a $\Phi$-space if for each entourage $V \in \mathbb{N}$, there exists a $\Phi$-mapping $T: X \rightarrow 2^{X}$ such that $\mathcal{G}_{T} \subset V$. Following the conceptions of the abstract convex space and the $\Phi$-space, we are easy to know that a bounded metric space $M$ is an important example of the abstract convex space, and if $X_{1} \subset X$ and $\mathcal{C}_{1}=\left\{C \cap X_{1}: C \in \mathcal{C}\right\}$, then $\left(X_{1}, \mathcal{C}_{1}\right)$ is also a $\Phi$-space.

Applying Theorem 2.5 of Amini et al. [7], we can deduce the following theorem in metric spaces.

Theorem 2.10. Let $X$ be a nonempty subadmissible subset of a metric space $(M, d)$. If $T \in$ $\operatorname{KKM}(X, X)$ is compact, then for each $r>0$, there exists $x_{r} \in X$; such that $B\left(x_{r}, r\right) \cap T\left(x_{r}\right) \neq \phi$.

Proof. Consider the family $\mathcal{C}$ of all subadmissible subsets of $M$ and for each $r>0, x \in X$, we set $V_{r}[x]=B(x, r)$. Let

$$
\mathbb{N}=\left\{V_{r} \mid V_{r}=\cup_{x \in M}\left\{(x, y): y \in V_{r}[x], r>0\right\}\right\} .
$$

Then $\mathbb{N}$ is a basis of a uniformity of $X$. For each $V_{r} \in \mathbb{N}$, we define two set-valued mappings $G, F: X \rightarrow 2^{X}$ by $G(x)=T(x)=V_{r}[x]$ for each $x \in X$. Then we have

(i) for each $x \in X, a d_{\mathcal{C}}(G(x))=a d_{\mathcal{C}}\left(V_{r}[x]\right)=V_{r}[x]=T(x) \subset V_{r}[T(x)]$;

(ii) $X=\cup_{x \in X} \operatorname{int}^{-1}(x)$.

So, $G$ is a companion mapping of $F$. This implies that $F$ is a $\Phi$-mapping such that $\mathcal{G}_{F} \subset V_{r}$. Therefore, $(X, \mathcal{C})$ is a $\Phi$-space.

Now we let $s: X \rightarrow X$ be an identity mapping, all of the the conditions of Theorem 2.5 of Amini et al. [7] are fulfilled, and we can obtain the results. 
Applying Theorems 2.1 and 2.10, we can conclude the following fixed point theorems.

Theorem 2.11. Let $X$ be a nonempty bounded subadmissible subset of a metric space $(M, d)$. If $T \in$ $\operatorname{KKM}(X, X)$ is a weaker Meir-Keeler type $\psi$-set contraction with for each $t \in \mathfrak{R}_{+},\left\{\psi^{n}(t)\right\}_{n \in \mathbb{N}}$ is noincreasing, and closed with $\overline{T(X)} \subset X$, then $T$ has a fixed point in $X$.

Theorem 2.12. Let $X$ be a nonempty bounded subadmissible subset of a metric space $(M, d)$. If $T \in K K M(X, X)$ is a generalized comparison (comparison) type $\psi$-set contraction and closed with $\overline{T(X)} \subset X$, then $T$ has a fixed point in $X$.

\section{References}

[1] B. Knaster, C. Kuratowski, and S. Mazurkiewicz, "Ein Beweis des Fixpunksatzes fur n-dimensionale Simplexe," Fundamenta Mathematicae, vol. 14, pp. 132-137, 1929.

[2] K. Fan, "A generalization of Tychonoff's fixed point theorem," Mathematische Annalen, vol. 142, pp. 305-310, 1961.

[3] A. Amini, M. Fakhar, and J. Zafarani, "KKM mappings in metric spaces," Nonlinear Analysis: Theory, Methods \& Applications, vol. 60, no. 6, pp. 1045-1052, 2005.

[4] T.-H. Chang and C.-L. Yen, "KKM property and fixed point theorems," Journal of Mathematical Analysis and Applications, vol. 203, no. 1, pp. 224-235, 1996.

[5] A. Meir and E. Keeler, "A theorem on contraction mappings," Journal of Mathematical Analysis and Applications, vol. 28, pp. 326-329, 1969.

[6] I. A. Rus, Fixed Point Theorems for Multivalued Mappings in Complete Metric Spacs, Cluj University Press, Cluj-Napoca, Romania, 2001.

[7] A. Amini, M. Fakhar, and J. Zafarani, "Fixed point theorems for the class S-KKM mappings in abstract convex spaces," Nonlinear Analysis: Theory, Methods \& Applications, vol. 66, no. 1, pp. 14-21, 2007. 